\title{
Mối quan hệ giữa xuất khẩu và năng suất lao động của doanh nghiệp
}

\section{Relationship between exporting and productivity of the enterprises}

\author{
Phạm Đình Long ${ }^{1 *}$, Nguyễn Chí Tâm²
}

${ }^{1}$ Trường Đại học Mở Thành Phố Hồ Chí Minh, Việt Nam

${ }^{2}$ Cục thuế Thành phố Hồ Chí Minh, Việt Nam

*Tác giả liên hệ, Email: long.pham@ou.edu.vn

\section{THÔNG TIN}

DOI: 10.46223/HCMCOUJS. econ.vi.13.2.512.2018

Ngày nhận: 28/11/2017

Ngày nhận lại: 04/04/2018

Duyệt đăng: 04/05/2018

Tì khóa:

hiệu ứng tự chọn lọc, học từ xuất khẩu, năng suất doanh nghiệp, quy mô của doanh nghiệp

Keywords:

self-selection, learning-byexport, firm productivity, firm size

\section{TÓM TÁT}

Bài viết này nghiên cứu mối quan hệ giữa xuất khẩu và năng suất lao động của doanh nghiệp có qui mô vừa và nhỏ tại Việt Nam với dữ liệu được sử dụng là dữ liệu bảng về hoạt động doanh nghiệp trong 10 năm từ 2002-2012. Để phân tích mối quan hệ trên, hai lý thuyết được sử dụng là tự chọn lọc (self-selection) và học từ xuất khẩu (learning-by-export). Nghiên cứu cho thấy tác động của hiệu ứng tự chọn lọc quyết định việc doanh nghiệp tham gia hay không tham gia vào thị trường xuất khẩu và hiệu ứng học từ xuất khẩu tác động đến năng suất của doanh nghiệp khi tham gia vào thị trường xuất khẩu, đồng thời giúp doanh nghiệp nâng cao hoạt động kinh doanh, cải thiện về vốn đầu tư và quy mô của doanh nghiệp.

\begin{abstract}
This paper investigates the relationship between exporting and productivity of Vietnam small and medium enterprises using firm level panel dataset in the period 2002-2012. To analyze this relationship, we apply two approaches, namely self-selection and learning-by-export. Our study reveals that self-selection determines whether or not the SMEs join the export market and the learning-by export affects the firm productivity when they participate in the export market, and it also improves their business activities, investment capital and scales up the business.
\end{abstract}

\section{Giới thiệu}

Mục tiêu của nghiên cứu là phân tích mối quan hệ giữa xuất khẩu và năng suất lao động của doanh nghiệp (DN) bằng việc vận dụng 2 lý thuyết (i) Lý thuyết về Sự tự chọn lọc (SelfSelection, SS), (ii) Lý thuyết về Học hỏi bằng việc xuất khẩu (Learning-By-Exporting, LBE) 
làm nền tảng phân tích mối quan hệ trên. Những DN hoạt động hiệu quả và có năng suất cao hơn những DN khác của cùng quốc gia và hoạt động trong cùng ngành, có xu hướng mở rộng thị trường kinh doanh của mình ra nước ngoài thông qua hoạt động xuất khẩu. Việc DN có năng suất cao hơn các $\mathrm{DN}$ khác và có xu hướng tìm đến thị trường mới để mở rộng hoạt động (Bernard \& Jensen, 1999; Bernard \& Wagner, 1997; Clerides, Lach, \& Tybout, 1998) được gọi là Sự tự chọn lọc (Self-selection, SS). Sau khi tham gia vào thị trường xuất khẩu, thị trường xuất khẩu sẽ tác động đến $\mathrm{DN}$ buộc $\mathrm{DN}$ phải thay đổi để thích nghi, đồng thời thông qua việc cạnh tranh của các $\mathrm{DN}$ khác trong cùng lĩnh vực giúp cho các $\mathrm{DN}$ tham gia xuất khẩu cải thiện được lợi nhuận của mình (Aw, Chung, \& Roberts, 2000; Bernard \& Jensen, 1999; Bernard \& Wagner, 1997). Việc DN tham gia vào thị trường xuất khẩu và cải thiện được năng suất của mình đồng thời lợi nhuận tăng lên được gọi là Học hỏi bằng việc xuất khẩu (Learning-ByExporting, LBE). Để minh chứng cho hai lý thuyết nói trên, đã có rất nhiều nghiên cứu đã được thực hiện tại nhiều quốc gia như Mỹ (Bernard \& Jensen, 1999), Anh (Girma, Greenway, \& Kneller, 2004) đến các quốc gia đang phát triển như Đài Loan (Liu, Tsou, \& Hammitt, 1999) đều đi đến cùng kết luận là năng suất lao động của DN là yếu tố tác động để DN quyết định tham gia xuất khẩu hay còn được gọi là tác động của hiệu ứng Tự chọn lọc (self-selection, SS) và sau khi tham gia xuất khẩu thì những tác động từ thị trường xuất khẩu hay còn gọi là hiệu ứng Học bằng xuất khẩu (learning-by-exporting, $\mathrm{LBE}$ ) giúp cho năng suất lao động của $\mathrm{DN}$ tăng cao hơn và đồng thời giúp $\mathrm{DN}$ phát triển hơn về qui mô và vốn so với trước khi tham gia xuất khẩu.

Trong phạm vi nghiên cứu, chúng tôi phân tích tác động của xuất khẩu đến năng suất lao động của Doanh nghiệp vừa và nhỏ (SMEs) với dữ liệu khảo sát về hoạt động kinh doanh trong vòng 10 năm (2002 - 2012). DN có qui mô vừa và nhỏ (SMEs), đây là một hình thức $\mathrm{DN}$ đặc thù của nền kinh tế Việt $\mathrm{Nam}$, những $\mathrm{DN}$ này có đặc điểm chung là có qui mô rất nhỏ và vốn đầu tư rất thấp, ngành nghề sản xuất đơn giản, không có hàm lượng công nghệ cao. Việc nghiên cứu tác động của xuất khẩu đến năng suất của SMEs và kết quả sẽ làm cơ sở để các SMEs có thể nhận thấy các vấn đề gây hạn chế và trở ngại cho họ trong việc tham gia xuất khẩu và có biện pháp cải thiện để hoạt động kinh doanh ngày càng phát triển hơn.

Phần còn lại của nghiên cứu được cấu trúc như sau: Phần 2 trình bày cách tiếp cận thực nghiệm của chúng tôi để thử nghiệm để tự lựa chọn và xác định học tập bằng cách xuất khẩu, mô tả dữ liệu và phân tích kết quả nghiên cứu, phần 3 là kết luận.

\section{Cơ sở lý thuyết và Phương pháp nghiên cứu}

\subsection{Co' sở lý thuyết}

Thương mại quốc tế (TMQT) hay còn gọi là xuất khẩu là quá trình trao đổi hàng hóa, dịch vụ giữa các quốc gia thông qua buôn bán nhằm mục đích kinh tế (Krugman, Obstfeld, \& Melitz, 2011). Các nhà nghiên cứu trước đây như Adam Smith, David Ricardo và HecksherOhlin đã đưa ra các lý thuyết và chứng minh được rằng xuất khẩu là một hoạt động có lợi cho sự phát triển của kinh tế các quốc gia mà cụ thể hơn là các $\mathrm{DN}$ trong các quốc gia đó sẽ có cơ hội phát triển qui mô sản xuất và kinh doanh hòng kiếm lợi nhuận cao hơn.

Self-selection (SS) qua các nghiên cứu được mô tả như một nền tảng cơ bản cho mọi DN quốc nội trước quyết định bước vào thị trường thế giới. Khi mà thị trường trong nước đã 
không đủ để cho DN khai thác và lợi nhuận không tăng thêm hoặc tăng chậm thì việc nhắm đến một thị trường rộng lớn hơn là điều tất yếu. Các DN tại mỗi ngành sản xuất đều sẽ có xu hướng tìm đến thị trường phù hợp (Aw et al., 2000; Aw, Roberts, \& Winston, 2007), để xác định việc mình có thể tham gia vào thị trường thì $\mathrm{DN}$ khảo sát thị trường với những yêu cầu nhất định về chất lượng hàng hóa (Clerides et al., 1998), nguồn vốn và kỹ năng lao động của DN (Bernard \& Jensen, 1999) và những chi phí để tham gia (Bernard \& Wagner, 1997; Roberts \& Tybout, 1997). Những chi phí ban đầu này sẽ là rào cản ban đầu cho các DN cần phải vượt qua nên đòi hỏi $\mathrm{DN}$ phải có một nền tảng kinh doanh vững chắc ban đầu trước khi quyết định tham gia, nếu không có sự chuẩn bị nhất định thì việc thất bại tại thị trường xuất khẩu khi mà mức chi phí tham gia cao hơn so với lợi nhuận mang lại sẽ dẫn đến việc rút khỏi thị trường xuất khẩu của DN. Minh chứng cho việc này là nghiên cứu của Bernard và Jensen (1999) dẫn chứng việc rút khỏi thị trường xuất khẩu với số lượng hơn $15 \% \mathrm{DN}$ xuất khẩu trong năm nay sẽ ngừng xuất khẩu vào năm sau, và $10 \% \mathrm{DN}$ mới sẽ tham gia vào xuất khẩu theo số liệu điều tra DN tại Mỹ.

Theo Bernard và Wagner (1997), Bernard và Jensen (1999), Krugman và cộng sự (2011), đã nêu rằng Learning-by-Exporting (LBE) là "Kinh nghiệm, kiến thức nhận được khi tham gia thị trường xuất khẩu đến từ các đối thủ cạnh tranh và người mua sẽ giúp cho $\mathrm{DN}$ tăng trưởng và phát triển nhanh hơn những $\mathrm{DN}$ chỉ sản xuất và bán hàng trong thị trường nội địa". Hiệu ứng học từ xuất khẩu có được từ lan tỏa theo 2 chiều: theo chiều dọc là sự cạnh tranh của các đối thủ; theo chiều ngang là sự so sánh của khách hàng. Bernard và Wagner (1997) nói rằng sự cạnh tranh (theo chiều dọc) đến trực tiếp từ các DN thâm nhập thị trường quốc tế, từ đối thủ cạnh tranh, qua đó được tiếp xúc với sự cạnh tranh khốc liệt hơn và cạnh tranh theo chiều ngang đến từ các khách hàng nước ngoài có thể muốn cải thiện quy trình công nghệ bằng cách cung cấp các thiết kế sản phẩm, thông số kỹ thuật và hỗ trợ kỹ thuật. Như vậy, "Kinh nghiệm, kiến thức nhận được khi tham gia thị trường xuất khẩu đến từ các đối thủ cạnh tranh và người mua sẽ giúp cho $\mathrm{DN}$ tăng trưởng và phát triển nhanh hơn những $\mathrm{DN}$ chỉ sản xuất và bán hàng trong thị trường nội địa". Kiến thức tích lũy được trong khu vực xuất khẩu chủ yếu là kết quả của các hoạt động học hỏi thông qua hoạt động xuất khẩu. Học hỏi thông qua xuất khẩu liên quan đến lợi ích mà nhà xuất khẩu thu được từ những kiến thức của người mua, khi người mua đưa ra những ý kiến đóng góp về chất lượng sản phẩm, giúp DN cải tiến qui trình sản xuất để sản phẩm phù hợp với nhu cầu của người mua (Bernard \& Jensen, 1999; Bernard \& Wagner, 1997; Krugman et al., 2011). Helpman và Krugman (1985) khẳng định lại quan điểm trên, nghiên cứu đã chỉ ra rằng xuất khẩu có thể làm tăng năng suất $\mathrm{DN}$ nhờ hiệu quả kinh tế theo quy mô. Tăng cường xuất khẩu cho phép DN mở rộng qui mô, giảm chi phí và có thể cho kết quả đạt năng suất cao hơn nữa.

\subsubsection{Kiểm định hiệu ứng Self-selection}

Clerides và cộng sự (1998) đề xuất hai giả thuyết có thể kiểm chứng một cách nhất quán với sự lựa chọn của các $\mathrm{DN}$ vào các thị trường xuất khẩu: (i) Nếu $\mathrm{DN}$ đang là xuất khẩu và có sự vượt trội về năng suất lao động giữa kỳ hiện tại so với kỳ trước thì DN sẽ tiếp tục xuất khẩu (ii) Nếu DN đang xuất khẩu nhưng năng suất lao động của kỳ hiện tại thấp hơn kỳ trước thì DN sẽ rút ra khỏi thị trường xuất khẩu. 
Năng suất lao động (NSLĐ) được định nghĩa là tỷ lệ giữa sản phẩm đầu ra (output) với sản phẩm đầu vào được sử dụng (Freeman, 2008), đối với đầu ra được xác định là tổng hàng hóa hoặc dịch vụ được làm ra bởi lực lượng lao động, được tính bằng GDP hoặc GVA, đối với đầu vào là Lao động thì được tính bằng Tổng giờ công lao động hoặc tất cả công nhân đang lao động (total employment).

Để kiểm tra hai giả thuyết của Clerides đề xuất, chúng tôi sử dụng NSLĐ (Freeman, 2008) làm chuẩn mực so sánh, năng suất lao động được tính bằng phần giá trị tăng thêm trên tổng nhân công của DN. DN có sự thay đổi về năng suất giữa kỳ hiện tại và kỳ trước được xác định bằng cách tính hiệu của năng suất lao động giữa 2 kỳ, nếu kết quả là tích cực (>0) và ngược lại là không có sự gia tăng về năng suất $(\leq 0)$. Mô hình kiểm định đề xuất tại phương trình 1.

$$
\begin{gathered}
\text { export }_{i j t}=\alpha_{1} \text { prodShock }_{i j t-1}+\alpha_{2} \text { aLabour }_{i j t-1}+\alpha_{3} \text { labourProd }_{i j t-1} \\
+\alpha_{4} \text { capLabour }_{i j t-1}+c_{i t}+s_{j}+e_{i j t}
\end{gathered}
$$

Với các biến như sau:

\section{Bảng 1}

\begin{tabular}{llc}
\hline \multicolumn{1}{c}{ Tên biến } & \multicolumn{1}{c}{ Ý nghĩa } & Kỳ vọng dấu \\
\hline export & 1: Tham gia; & + \\
& 0: Không tham gia. & + \\
prodShock & Tăng năng suất giữa năm hiện tại và năm & \\
& trước: & \\
& 1: Nếu > 0; & + \\
& 0: Nếu < 0. & + \\
aLabour & Quy mô DN & + \\
labourProd & Năng suất lao động & + \\
capLabour & Tỷ lệ vốn trên lao động & \\
$\mathrm{c}_{\mathrm{it}}$ & Loại hình chủ sở hữu & \\
$\mathrm{s}_{\mathrm{j}}$ & Ngành, nghề của DN (VSIC) & \\
$\mathrm{e}_{\mathrm{ijt}}$ & Sai số & \\
\hline
\end{tabular}

Nguồn: Kết quả phân tích dữ liệu của nhóm nghiên cứu

Xác định các biến trong mô hình như sau:

- Biến export là biến dummy đại diện cho hoạt động xuất khẩu hoặc không có xuất khẩu của SMEs (Krugman et al., 2011);

- Biến prodShock là biến dummy, được xác định bằng Giá trị gia tăng (Value added) trên Tổng số lao động (Bernard \& Jensen, 1999; Bernard \& Wagner, 1997; Clerides et al., 1998) đại diện cho sự thay đổi năng suất lao động (Freeman, 2008) được xác định từ việc so sánh giữa NSLĐ (Freeman, 2008) kỳ trước và kỳ hiện tại, nếu NSLĐ của kỳ hiện tại cao hơn kỳ trước thì prodShock $=1$ và ngược lại prodShock $=0$;

- Biến aLabour đại diện cho tổng lao động của SMes (Aw et al., 2000; Bernard \& Jensen, 1999; Bernard \& Wagner, 1997; Freeman, 2008), xác định tổng nhân công thuê trong thời gian đang hoạt động; 
- Biến labourProd (labour productivity) là Năng suất lao động được tính bằng Giá trị tăng thêm (value added) trên Tổng nhân công (aLabour). Giá trị tăng thêm (Value Added) = Tổng sản phẩm (production) - Tổng chi phí nguyên vật liệu (raw materials) - Tổng chi phí gián tiếp (indirect cost) (Bernard \& Jensen, 1999; Bernard \& Wagner, 1997);

- Biến capLabour: Là tỷ lệ giữa vốn (capital) và tổng lao động (aLabour), capLabour = Vốn/Tổng lao động. Trong đó, Vốn được xác định bằng tổng tài sản hữu hình của $\mathrm{DN}$ (physical assets).

\subsubsection{Kiểm định hiệu ứng Learning-By-Export}

Chúng tôi sử dụng hướng tiếp cận việc kiểm định hiệu ứng Learning-By-Exporting được đề xuất bởi Bigsten và cộng sự (2004), Fernandes và Isgut (2005) và Van Biesebroeck (2005). Mô hình được đề xuất tại phương trình 2.

$$
\ln L B P_{i t}=\beta_{0} \operatorname{lnV}_{i t-1}+\beta_{1} \text { export }_{i t-1}+\beta_{2} \text { yearExp }_{i t-1}+\beta_{3} \operatorname{lnExRevenue}{ }_{i t}+\varphi_{1} Z_{1 i t}+\varphi_{2} Z_{2 i t}+s_{j}+e_{i t}(2)
$$

- $\operatorname{lnLBP}$ : Logarit (Năng suất lao động);

- export: Biến dummy có giá trị [0 1];

- Số năm kinh nghiệm yearExp (year experience) được xác định bằng cách tính từ thời điểm thực hiện khảo sát trừ cho năm $\mathrm{DN}$ bắt đầu thực hiện xuất khẩu, yearExp = Năm khảo sát - Năm bắt đầu +1 , biến có giá trị từ $[0 \ldots \mathrm{n}]$;

- Lợi nhuận từ xuất khẩu $\operatorname{lnExRevenue~xác~định~bằng~tỷ~lệ~lợi~nhuận~từ~xuất~khẩu~so~}$ với Tổng lợi nhuận DN đạt được trong năm (Baldwin \& Gu, 2003; Clerides et al., 1998; Van Biesebroeck, 2005);

- $\mathrm{Z}_{1}$ : Đại diện cho 2 yếu tố sản xuất là Vốn và Lao động, tính bằng logarit (capital) và logarit (aLabour);

- $\mathrm{Z}_{2}$ : Các biến trong mô hình SS được tính bằng logarit, gồm: export, labourProd, alabour, capLabour, được lấy ở trễ 2;

- s: Ngành, nghề hoạt động của DN;

- e: Sai số của mô hình.

Mô hình LBE được kiểm định nội sinh bằng phương pháp GMM, áp dụng cách thực hiện của Blundell và Bond (1998) là kiểm định biến phụ thuộc bị nội sinh bằng việc sử dụng biến công cụ là các biến dùng trong mô hình để hỗ trợ.

\subsection{Kết quả nghiên cúu}

\section{Bảng 2}

Kết quả kiểm định của mô hình Self-selection

\begin{tabular}{|l|c|l|}
\hline \multicolumn{1}{|c|}{ Biến mô hình } & FEM & \multicolumn{1}{|c|}{ Tên các biến trong mô hình } \\
\cline { 2 - 2 } & export & \\
\hline prodShock $_{\mathrm{t}-1}$ & $2.175^{* * *}$ & Cú shock năng suất \\
\hline aLabour $\mathrm{t}-1^{*}$ & 0.000713 & Tổng nhân công (qui mô) \\
\hline capLabour $_{\mathrm{t}-1}$ & 0.000508 & Tỷ lệ vốn trên lao động \\
\hline labourProd $_{\mathrm{t}-1}$ & -0.000453 & Năng suất lao động \\
\hline
\end{tabular}




\begin{tabular}{|c|c|c|}
\hline \multirow{2}{*}{ Biến mô hình } & FEM & \multirow{2}{*}{ Tên các biến trong mô hình } \\
\hline & export & \\
\hline gOwner $_{i=1}$ & -0.0555 & Nhóm loại hình chủ sở hữu \\
\hline improveGoods $\mathrm{s}_{\mathrm{i}=1}$ & 0.493 & Cải thiện chất lượng sản phẩm \\
\hline gIndustry $\mathrm{i}=2$ & 0.474 & Nhóm ngành nghề xuất khẩu \\
\hline \multicolumn{3}{|c|}{$* * * p<0,01, * * p<0,05, * p<0,1$} \\
\hline \multicolumn{3}{|c|}{$\begin{array}{l}\text { Mô hình trên đã kiểm soát tư tuơng quan và phương sai sai số thay đổi bằng ước lương } \\
\text { vưng cho sai số chuấn }\end{array}$} \\
\hline
\end{tabular}

Nguồn: Tính toán của tác giả

Với kết quả mô hình trên, có những phân tích sau:

- prodShock có tác động cùng chiều như kỳ vọng đến biến phụ thuộc là export. Như vậy, việc thay đổi năng suất lao động kỳ sau cao hơn kỳ trước có ý nghĩa quyết định đến việc gia nhập vào thị trường xuất khẩu của $\mathrm{DN}$. Như giả thuyết ban đầu đã đề cập đến, đối với $\mathrm{DN}$ có năng suất vượt trội hơn những $\mathrm{DN}$ cùng ngành thì sẽ đi đến quyết định gia nhập thị trường xuất khẩu.

- Đối với số nhân công (aLabour) và tỷ lệ vốn trên nhân công (capLabour) có tác động cùng chiều với biến phụ thuộc, điều này thể hiện rằng việc bước vào thị trường xuất khẩu ban đầu là chủ yếu do sự gia tăng về năng suất lao động, vốn và qui mô (tổng lao động) trong kỳ trước gần như không thay đổi (hệ số thấp). Tuy nhiên, với thời gian dài thì việc tăng về qui mô và đầu tư về vốn sẽ là điều tất yếu khi tham gia vào xuất khẩu, điều này phù hợp với giả thuyết về hiệu ứng LBE như đã định nghĩa ở trên.

- Đối với năng suất lao động (labourProd) có tác động ngược chiều so với kỳ vọng, nhưng với hệ số coef thấp thì tác động không đáng kể đến quyết định gia nhập xuất khẩu.

- Đối với biến loại hình chủ sở hữu (gOwner) có tác động ngược chiều với biến phụ thuộc cho thấy rằng việc xuất khẩu có tác động mạnh việc chuyển đổi loại hình hoạt động của DN từ dạng kinh doanh nhỏ lẻ như hộ gia đình, DN tư nhân sẽ ngày càng giảm, thay vào đó các DN dạng hộ gia đình với vốn của một cá nhân đầu tư sẽ chuyển sang loại hình có qui mô cao hơn với vốn góp là cổ phần của nhiều nơi, như vậy qui mô và vốn đầu tư sẽ tăng theo.

- Đối với cải thiện chất lượng sản phẩm (improveGoods) có tác động cùng chiều, tuy không đủ ý nghĩa thống kê nhưng cũng cho thấy rằng trong thời gian đầu gia nhập thị trường, DN không có sự thay đổi về chất lượng sản phẩm, nhưng có thể trong tương lai sẽ có sự cải thiện.

- Đối với biến ngành nghề (gIndustry) có tác động cùng chiều với biến phụ thuộc, cho thấy ngành nghề về khai thác tài nguyên và sản xuất máy móc có xu hướng xuất khẩu nhiều hơn so với các ngành khác. 


\section{Bảng 3}

Kết quả mô hình Learning-By-Export

\begin{tabular}{|c|c|c|}
\hline \multirow{2}{*}{ Biến mô hình } & SGMM & \multirow{2}{*}{ Tên biến } \\
\hline & $\operatorname{lnLBP}$ & \\
\hline $\ln \mathrm{VA}_{\mathrm{t}-1}$ & -0.0121 & Giá trị tăng thêm \\
\hline L.export & $-0.137^{*}$ & Có hoạt động xuất khẩu \\
\hline lnCap & 0.0183 & Vốn của DN \\
\hline lnLabour & $-0.884 * * *$ & Tổng nhân công \\
\hline lnExRevenue & $0.898 * * *$ & Lợi nhuận từ xuất khẩu \\
\hline yearExp $\mathrm{t}_{\mathrm{t}-1}$ & $0.0213 * *$ & Số năm kinh nghiệm xuất khẩu \\
\hline Export $_{\mathrm{t}-2}$ & -0.0756 & Có hoạt động xuất khẩu \\
\hline $\operatorname{lnLBP} \mathrm{t}_{-2}$ & $-0.0387 * *$ & Năng suất lao động \\
\hline $\operatorname{lnLabour}{ }_{t-2}$ & 0.0232 & Tổng nhân công \\
\hline $\operatorname{lnCL} \mathrm{L}_{\mathrm{t}-2}$ & $0.0462 *$ & Tỷ lệ vốn trên lao động \\
\hline gIndustry $\mathrm{i}=2$ & 0.0892 & $\begin{array}{l}\text { Nhóm ngành sản xuất (1: NPL và lương } \\
\text { thực; } 2 \text { : Tài nguyên) }\end{array}$ \\
\hline AR (1) & $\mathbf{0 , 0 7 8}$ & $<0,1$ \\
\hline AR (2) & $\mathbf{0 , 1 0 7}$ & $>0,1$ \\
\hline Hansen & $\mathbf{0 , 9 1 3}$ & $>0,1$ \\
\hline
\end{tabular}

Nguồn: Tính toán của tác giả

Với kết quả kiểm định của $\mathrm{AR}(1)$ và $\mathrm{AR}$ (2) và Hansen của phương pháp là SGMM (System GMM) với hệ số Arellano-Bond và Hansen đạt mức giá trị phù hợp, kết luận mô hình không có hiện tượng nội sinh.

Với kết quả mô hình như trên, có những nhận xét như sau:

- Việc xuất khẩu của kỳ trước có hiệu quả với DN, qui mô, năm kinh nghiệm trên thị trường xuất khẩu và lợi nhuận từ hoạt động xuất khẩu đều tăng theo.

- Tại thời điểm DN bắt đầu xuất khẩu thì qui mô không thay đổi (biến $\operatorname{lnLabour}$-2). Tuy nhiên, sau khi đã tham gia vào xuất khẩu, đến thời điểm hiện tại thì qui mô của $\mathrm{DN}$ có xu hướng tăng cao hơn so với thời điểm bắt đầu xuất khẩu.

Lợi nhuận từ hoạt động xuất khẩu tăng, đồng thời số năm kinh nghiệm trong xuất khẩu cũng tăng theo, chứng tỏ xuất khẩu làm tăng lợi nhuận và tích lũy kinh nghiệm hoạt động trên thị trường xuất khẩu.

\section{Kết luận}

Kết quả trên cho thấy, tác động của xuất khẩu đến năng suất của DN là có hiệu quả tích cực, phù hợp với lý thuyết và các nghiên cứu trước. Kết quả đã trả lời được câu hỏi là xuất khẩu tác động đến năng suất của $\mathrm{DN}$ và ngược lại, năng suất dưới tác động của xuất khẩu ngày càng tăng sẽ là động lực giúp DN tiếp tục duy trì việc xuất khẩu. 
Việc một DN muốn tham gia vào thị trường xuất khẩu cần phải có sự chuẩn bị về vốn và qui mô của $\mathrm{DN}$. Khi tham gia vào thị trường xuất khẩu, có một sự cạnh tranh giữa những đối thủ từ quốc gia nhập khẩu và các hàng hóa khác cùng loại trên thị trường, ngoài ra còn có sự chọn lọc từ chính khách hàng với nhu cầu và thị hiếu đa dạng. Việc $\mathrm{DN}$ nổi trội hơn cùng ngành và qui mô cao hơn sẽ giúp cho $\mathrm{DN}$ có lợi thế về qui mô khi cạnh tranh với $\mathrm{DN}$ nước ngoài, trong thời gian đầu tham gia thị trường xuất khẩu, DN phải tốn một khoản chi phí nhất định cho những vấn đề sau: Chi phí thăm dò thị trường mới; Chi phí quảng cáo; Chi phí đầu tư mới cho sản phẩm. Những chi phí trên sẽ làm tăng giá thành của sản phẩm, nếu $\mathrm{DN}$ bán giá cao hơn giá mặt bằng chung của thị trường xuất khẩu thì hàng hóa sẽ không bán được và đồng nghĩa với việc lỗ. Do đó, lợi thế về qui mô sẽ giúp $\mathrm{DN}$ bù đắp lại phần chi phí phải bỏ ra khi sản xuất hàng hóa bán cho thị trường quốc tế, giúp giá thành cạnh tranh hơn, theo thời gian thì DN sẽ có chỗ đứng trong thị trường. Việc $\mathrm{DN}$ không có sự chuẩn bị về vốn và qui mô sẽ là một điểm hạn chế lớn với những chi phí và những vấn đề khác, và sẽ làm cho $\mathrm{DN}$ không thể tiếp tục duy trì xuất khẩu. Như vậy, một $\mathrm{DN}$ phải trở thành một $\mathrm{DN}$ hoạt động tốt hơn những $\mathrm{DN}$ trong cùng ngành để có thể tham gia và thị trường xuất khẩu, đồng thời tiếp tục duy trì và lớn mạnh hơn.

Sau khi tham gia vào thị trường xuất khẩu, dưới tác động của thị trường, vốn và qui mô của DN cũng tăng theo, đồng thời lợi nhuận và kinh nghiệm cũng có sự tiến bộ hơn. Tuy nhiên, theo thời gian, DN lại có xu hướng rút ra khỏi thị trường xuất khẩu hơn, mặc dù việc tham gia vào xuất khẩu thời gian đầu thật sự có hiệu quả trong việc cải thiện tình hình hoạt động của $\mathrm{DN}$. Tuy việc $\mathrm{DN}$ có xu hướng rút ra khỏi thị trường xuất khẩu do năng suất lao động giảm nhiều dù $\mathrm{DN}$ đã có sự vượt trội về qui mô và vốn như đã nêu ở trên. Khi xem xét lại môi trường kinh doanh của $\mathrm{DN}$ có thể nhận thấy, việc các SMEs tham gia vào xuất khẩu chủ yếu là các $\mathrm{DN}$ hộ gia đình, đây là loại hình DN không có qui mô lớn, vốn ít, ngành sản xuất đơn giản chủ yếu tập trung vào lĩnh vực khai thác tài nguyên và gia công sản phẩm.

Tách biệt vai trò của năng suất lao động đối với xuất khẩu và xuất khẩu đối với năng suất lao động đã cho một cái nhìn chi tiết hơn về vai trò của từng tác động. Năng suất lao động là yếu tố đầu tiên mà một $\mathrm{DN}$ phải đạt được, đây là nền tảng vững chắc giúp $\mathrm{DN}$ có thể tiến vào thị trường xuất khẩu và có đủ thời gian để thích nghi và xuất khẩu thật sự làm thay đổi hoạt động kinh doanh của $\mathrm{DN}$. Kết quả của mô hình cho thấy là các $\mathrm{DN}$ cần có các biện pháp điều chỉnh phù hợp trong chiến lược kinh doanh của mình để nhằm đạt mục tiêu là tham gia xuất khẩu. Việc tham gia vào xuất khẩu nhìn chung là có lợi cho hoạt động kinh doanh, sản xuất của $\mathrm{DN}$ trong nhiều phương diện. Thị trường xuất khẩu làm cho $\mathrm{DN}$ có cơ hội đổi mới và cải thiện hoạt động kinh doanh của mình, với kết quả mô hình cho thấy rằng, DN khi tham gia vào sẽ tích lũy được kinh nghiệm trong hoạt động xuất khẩu đồng thời cải thiện thu nhập từ xuất khẩu của mình. Ngoài ra, bản thân $\mathrm{DN}$ phải chú trọng việc đổi mới công nghệ và chất lượng sản phẩm để có thể tiếp tục duy trì hoạt động xuất khẩu của mình.

\section{Tài liệu tham khảo}

Arellano, M., \& Bond, S. R. (1991). Some tests of specification for panel data: Monte Carlo evidence and an application to employment equations. Review of Economic Studies, 58, 277-297. 
Aw, B., Chung, S., \& Roberts, M. (2000). Productivity and turnover in the export market: Micro-level evidence from Republic of Korea and Taiwan (China). World Bank Economic Review, 14(1), 65-90.

Aw, B., Roberts, M., \& Winston, T. (2007). Export market participation, investments in R\&D and worker training, and the evolution of firm productivity. The World Economy, 14(1), 83-104.

Aw, B., Roberts, M., \& Xu, D. Y. (2011). R\&D investments, exporting, and productivity dynamics. American Economic Review, 101(4), 1312-1344.

Baldwin, J., \& Gu, W. (2003). Export-market Paritcipation and productivity performance in Canadian manufacturing. Canadian Journal of Economics, 36(3), 634-657.

Bernard, A., \& Jensen, J. (1999). Exceptional exporter performance: Cause, effect or both? Journal of International Economics, 47(1), 1-25.

Bernard, A., \& Wagner, J. (1997). Exports and success in German manufacturing. Weltwirtschaftliches Archiv, 133(1), 134-147.

Bigsten, A., \& Gebreeyesus, M. (2009). Firm productivity and exports: Evidence from Ethiopian manufacturing. Journal of Development Studies, University of Gothenburg Sweden, 45(10), 1594-1614.

Bigsten, A., Collier, P., Dercon, S., Fafchamps, M., Gauthier, B., Gunning, J. W., ... Zeufack, A. (2004). Do African manufacturing firms learn from exporting? Journal of Development Studies, 40(3), 115-141.

Blalock, G., \& Gertler, P. (2004). Learning from exporting revisited in a less developed setting. Journal of Development Economics, 75, 397-416.

Blundell, R., \& Bond, S. (1998). Initial conditions and moment restrictions in dynamic panel data models. Journal of Econometrics, 87, 115-143.

Clerides, S. K., Lach, S., \& Tybout, J. R. (1998). Is learning by exporting important? Microdynamic evidence from Columbia, Mexico and Morocco. Quarterly Journal of Economics, 113(3), 903-947.

Fernandes, A., \& Isgut, A. (2005). Learning-by-doing, learning by exporting and productivity: Evidence from Colombia. Retrieved October 11, 2017, from https://elibrary.worldbank.org/doi/abs/10.1596/1813-9450-3544

Freeman, R. (2008). Labour productiviy indicators. Retrieved October 13, 2017, from http://www.oecd.org/employment/labour-stats/41354425.pdf

Giles, D. E. A, Giles, J. A., \& McCann, E. (1992). Causality, unit roots and exported growth: The New Zealand experience. Retrieved October 12, 2017, from https://ageconsearch.umn.edu/record/263698/files/canterbury-nz-053.pdf

Girma, S., Greenaway, D., \& Kneller, R. (2004). Does exporting increase productivity? A microeconometric analysis of matched firms. Review of International Economics, 12(5), 855-866.

Grossman, G. M., \& Helpman, E. (1990). Comparative advantage and long-run growth. American Economic Review, 80(4), 796-815. 
Hansen, L. P., (1982). Large sample properties of generalised method of moment estimators. Econometrica, 50, 1029-1054.

Helpman, E., \& Krugman, P. (1985). Market structure and foreign trade: increasing returns, imperfect competition, and the international economy. Cambridge, MA: MIT Press.

Krugman, P. R., Obstfeld, M., \& Melitz, M.J. (2011). International economics: Theory and policy (9th ed.). Boston, MA: Addison-Wesley.

Lileeva, A., \& Trefler, D. (2010). Improved access to foreign markets raises plant-level productivity... for some plants. Quarterly Journal of Economics, 125(3), 1051-1099.

Liu, J.-T., Tsou, M.-W., \& Hammitt, J. K. (1999). Export activities and productivity: Evidence from the Taiwan electronics industry. Review of World Economics, 135(4), 675-691.

Lu, X., \& White, H. (2014). Robustness check and robustness tests in applied econometrics. Journal of Econometrics, 178, 194-206.

Roberts, M., \& Tybout, J. (1997). The decision to export in Colombia: An empirical model of entry with sunk costs. American Economic Review, 87, 545-564.

Thirlwall, A. P. (2003). Trade, the balance of payments and exchange rate policy in developing countries. Retrieved October 14, 2017, from https://www.e-elgar.com/shop/gbp/tradethe-balance-of-payments-and-exchange-rate-policy-in-developing-countries9781843762294.html

Van Biesebroeck, J. (2005). Exporting raises productivity in sub-Saharan African manufacturing firms. Journal of International Economics, 67, 373-391.

Vu, H. V. (2012). Higher productivity in exporters: Self-selection, learning by exporting or both? Evidence from Vietnamese manufacturing SMEs. Retrieved October 15, 2017, from https://mpra.ub.uni-muenchen.de/40708/ 